\title{
Lepton pair production with only one charge available - neutrino
}

\author{
Ari Lehto \\ Aalto University, Espoo, Finland \\ cwmies@gmail.com
}

\begin{abstract}
According to observations leptons are always produced in pairs. Both particles can be electrically charged, or just one, in which case the charged particle is accompanied by a particle specific neutrino. The period doubling process in nonlinear dynamical systems creates stable pair structures from the Planck units. The electron-positron pair is one of the stable structures, and the rest mass, electric charge and magnetic moment can be accurately calculated by the period doubling process. That the pair structure is stable means, that e.g., an electron and a positron are always born together. In the muon decay one of the pair is charged, while the other remains chargeless because there is only one charge available. It is suggested in this article that neutrino is the chargeless part of the lepton pair.
\end{abstract}

Keywords: neutrino, period doubling, muon decay, elementary charge, electron mass, Planck units

\section{INTRODUCTION}

Subharmonic creation, called the period doubling phenomenon, is a universal property of nonlinear dynamical systems, as theoretically shown by M. Feigenbaum [1]. The period doubling phenomenon has been observed in various physical and chemical systems like lasers [2], plasmas [3], chemical reactions [4], planetary systems [5], stars [6], and galaxy redshifts [7]. It has also been shown that electron physical properties (mass, value of the elementary charge and magnetic moment), as well as the value of the fine structure constant, can be calculated from the corresponding Planck units by the period doubling phenomenon $[8,9,10,11]$ including the internal degrees of freedom in the system $[10$, $12]$.

\section{METHOD}

In this study the lepton production and decay processes are assumed nonlinear and examined using period doubling formulas applied to the relevant Planck units.

The Planck units are defined using $h$ instead of $h$-bar, because energy $E=h f=h /$ period, and the physical process is period doubling. Several other physical quantities can be derived from period, too [11]. The required Planck units are:

The Planck charge $q_{o}$

$$
q_{o}=\sqrt{4 \pi \varepsilon_{o} h c}
$$

$q_{o}=4.7013 \cdot 10^{-18}$ As.

The Planck mass

$$
m_{o}=\sqrt{\frac{h c}{G}}
$$

$m_{o}=5.4555 \cdot 10^{-8} \mathrm{~kg}$.

The Planck (mass) energy $E_{o}$

$$
E_{o}=m_{o} c^{2}
$$

$E_{o}=3.0603 \cdot 10^{22} \mathrm{MeV}$.

The rest energy (4) of the electron-positron pair structure produced by 3D period doubling is [11]: 


$$
E_{e^{+} e^{-}}=2^{-\frac{32+64+128}{3}} \cdot E_{o}=1.021 \mathrm{MeV}
$$

Integers $32=2^{5}, 64=2^{6}$ and $128=2^{7}$ indicate the number of period doublings, or energy halvings, in each orthogonal degree of freedom. Each degree of freedom contains energy according to $E=h /$ period, and the periods form a 3D periodic phase space structure. For the observer energy is not a $3 \mathrm{D}$ volume, but a scalar. The cube root in (4) returns the 3D periodic volume of the electron-positron pair to a characteristic period, which is the observable rest energy in this case [11]. The fractional exponents of two have been discovered in an earlier analysis of the experimental data [12].

The criterion for the superstability of the $n$ 'th period is defined by a subset of periods [1]:

$$
t_{n}=2^{2^{n}} \cdot t_{o}
$$

where $n$ is a positive integer and $t_{o}$ the fundamental period of the system. In equation (4) the three periods are of the form of (5) meaning that the stable $1.021 \mathrm{MeV}$ pair structure is always born.

The ratio of the Coulomb energies is expressed in (6). The geometric mean, or fourth root, of the periods indicates that the electrostatic part of the system has four degrees of freedom. The periods $(1,2,4,32)$ in (6) are superstable according to the stability condition (5).

$$
\left(\frac{q_{o}}{e}\right)^{2}=2^{9.75}=2^{\frac{39}{4}}=2^{\frac{1+2+4+32}{4}}
$$

Equation (6) yields for the elementary charge $e=1.6021 \cdot 10^{-19}$ As, which differs from the CODATA value by $0.003 \%$ [13]. No tuning in (4) and (6) is possible without tuning the related natural constants.

\section{RESULTS}

The period doubling process shows that the electrons and positrons are always born in pairs. This is also confirmed by experiments. Both members of the pair can be charged, or just one, as shown by the examples in Figure 1. The muon neutrino has been omitted from the muon decay chart. Electrically neutral gamma quantum produces $+/$-charged pairs, whereas in the muon decay only one charged

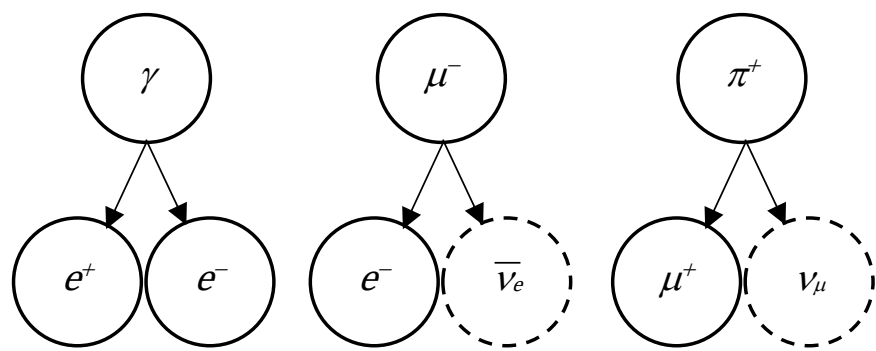

Figure 1. Lepton pair production. particle is produced because there is only one charge available. The pair is born anyway, but the other particle remains chargeless. The same applies to the tau, kaon, and pion decays into a muonneutrino pair. The $3 \mathrm{D}$ periodic structures of the pair are identical, and the absolute angular momentum of the chargeless particle is the same as that of the charged particle, otherwise the neutrino could not produce an electron in the reverse process. The $4 \mathrm{D}$ periodic structure of the Coulomb energy is independent of the periodic 3D mass structure, as can be seen from the different number of doublings in (4) and (6).

An interesting observation is that the charged particle of the pair is always the mass carrier. Consequently, the chargeless particle (neutrino) is massless. The omitted muon (anti)neutrino (Fig.1) is the chargeless and massless periodic 3D 'frame' or 'skeleton' of the muon left over at the charge transfer to the electron structure. The $\mu^{-}$is always balanced by the $\mu^{+}$of the original $\mu^{+} \mu^{-}$pair. Conservation of momentum requires that the massless particle flies away at the speed of light carrying the angular momentum with it. Note that 'chargeless' is not the same as 'neutral'. A neutron has negative surface charge and positive inside, and it decays into a proton, electron, and electron antineutrino (the pair). 
The reverse processes have also been observed, where the electron (anti) neutrinos always produce electrons, muon neutrinos muons and tau neutrinos tau particles. This is because the neutrino has the same 3D periodic structure as the charged particle of the pair. In this case the chargeless structure becomes charged and obtains the particle properties (mass, charge, magnetic moment).

\section{DISCUSSION}

Harmonic frequency generation in nonlinear systems is a well-known phenomenon. If $f_{o}$ is the fundamental frequency, then the harmonic frequencies are $f_{n}=n \cdot f_{o}$, where $n=2,3,4,5, \ldots$ Applications can be found for instance in radio- and laser technologies. In a linear system there are no harmonic frequencies.

It is less widely known that subharmonics may be generated, too. The process is called period doubling or frequency halving, which M. Feigenbaum proved universal property of nonlinear dynamical systems [1]. The subharmonics $f_{n}=2^{-n} \cdot f_{o}$ stabilize (or become chaotic) in the course of time. This phenomenon effectively reduces the large Planck energy to energies found in the particle world, when the Planck energy is taken as the fundamental energy for an elementary particle system.

There are three kinds of a neutrino, specific to the three types of the leptons. The essence of neutrinos is largely unknown because they are overwhelmingly difficult to observe. It is known that their speed is remarkably close to $c$, and that they may, or may not, have a small mass.

According to (4) the period doubling process produces stable electron-positron pairs, which means that an electron (or positron) can never be produced alone. If there is only one charge available for the pair, as in the muon decay, then the other particle inevitably remains chargeless and the corresponding neutrino is created. Experiments confirm that the same applies to the muons and tau particles produced in various particle processes. The chargeless particle is now assumed to be the neutrino.

The identical 3D (periodic) structures of the pair are crucial for the reverse process to take place, where electron antineutrinos always produce structurally identical electrons. The pair production also means that neutrinoless beta (or +beta) decay is impossible.

The charged particle of the pair is always the mass carrier. When a massless frame (neutrino) becomes charged, it gains mass. This means that the charge has the mass property because the $3 \mathrm{D}$ periodic frame is massless.

An interesting question is the possible entanglement of the particle-neutrino pair because it is the pair that is stable. In the neutrino oscillation scenario, the flavour of a neutrino can change meaning that e.g., the 3D periodic structure of an electron antineutrino can transform into the structure of a muon antineutrino. If the entanglement is strong, then the partner electron should transform into a muon.

\section{CONCLUSIONS}

Period doubling, or frequency halving, is an experimentally and theoretically well-established property of nonlinear dynamical systems. It offers a natural way to reduce the large Planck energy to the energy levels of the particle world. The reduction by period doubling of the Coulomb energy of the Planck charge creates the elementary charge and correspondingly the electron-positron pair rest energy from the Planck mass. The periodic structures have internal degrees of freedom, which must be considered. In this article it is shown that the periodic structure of an electron-positron pair belongs to a subset of stable periods. The pair can contain $+/$ - charged particles, or only one charged particle. The charged and chargeless particles have identical 3D periodic structures, and it is concluded that the chargeless particle is the particle specific neutrino. The charged particle of the pair is the mass carrier, so the chargeless particle must be massless in principle. In the reverse process the massless particle (neutrino) takes on electric charge and becomes massive. Therefore, it is the electric charge that has the mass property. 
Particles decaying into leptons always produce lepton pairs, either charged or one uncharged. Conclusions concerning the electron-positron pair creation can be extended to cover all particle decays into lepton pairs.

The period doubling approach makes it possible to calculate electron mass and electric charge from the Planck units thus reducing the number of natural constants by two, and it may give new insight into the origin and essence of the neutrino as the chargeless member of the lepton pair.

\section{ACKNOWLEDGMENTS}

I would like to express my special thanks of gratitude to Dr's T. Suntola, H. Sipilä, T. Kallio-Tamminen, M. Hyvönen-Dabek and J. Dabek for many insightful discussions and comments.

\section{REFERENCES}

[1] M. J. Feigenbaum, Universal behavior in nonlinear systems, Physica 7D, (1983), 16-39

[2] Simpson T. B., Liu J. M., Gavrielides A., Kovanis V. and Alsing P. M., Period-doubling route to chaos in a semi-conductor laser subject to optical injection, Appl. Phys. Lett. 64, 3539, 1994

[3] Cheung P. Y. and Wong A. Y., Chaotic behavior and period doubling in plasmas, Phys. Rev. Lett. 59, 551, 3, August 1987

[4] Bradley Marts, David J. W. Simpson, Aric Hagberg, and Anna L. Lin, Period doubling in a periodically forced Belousov-Zhabotinsky reaction, Physical Review E76, 026213, 2007

[5] Batygin K., Morbidelli A., "Onset of Secular Chaos in Planetary Systems: Period Doubling \& Strange Attractors", arXiv: 1106.2590

[6] R. Smolec, I. Soszy' nski, P. Moskalik, A. Udalski, M. K. Szymá nski, M. Kubiak, G. Pietrzy'nski, Ł. Wyrzykowski, K. Ulaczyk, R. Poleski, S. Kozłowski and P. Pietrukowicz, Discovery of period doubling in BL Herculis stars of the OGLE survey. Observations and theoretical models, R. Mon. Not. R. Astron. Soc. 419, 2407-2423, 2012, doi: 10.1111/j.1365-2966.2011.19891.x

[7] William G. Tifft, Redshift - Key to Cosmology, AlphaGraphics, ISBN 978-0-9862619-0-9, 2014 https://williamtifft.wordpress.com

[8] Lehto, A. Period Doubling Phenomenon as the Origin of Electron Properties. Preprints 2020, 2020060076

[9] Sipilä, H.; Lehto, A. Fundamental Constants in Time from the Big-Bang. Preprints 2020, 2020070239, doi: 10.20944/preprints202007.0239.v1

[10] Ari Lehto, Periodic time and the stationary properties of matter, Chin. J. Phys., 28, 3, 215-235, June 1990

[11] Ari Lehto: On the Planck Scale and Properties of Matter, International Journal of Astrophysics and Space Science. Special Issue: Quantum Vacuum, Fundamental Arena of the Universe: Models, Applications and Perspectives. Vol. 2, No. 6-1, pp. 57-65, 2015, doi:10.11648/j.ijass.s.2014020601.17

[12] Lehto A., "On the Planck Scale and Properties of Matter", Nonlinear Dynamics, 55, 3, 279-298, February 2009

[13] https://physics.nist.gov/cuu/Constants/ 